\title{
High serum concentrations of autoantibodies to HSP47 in nonspecific interstitial pneumonia compared with idiopathic pulmonary fibrosis
}

\author{
Tomoyuki Kakugawa ${ }^{1}$, Shin-ichi Yokota ${ }^{2}$, Hiroshi Mukae ${ }^{* 1}$, Hiroshi Kubota ${ }^{4}$, \\ Noriho Sakamoto ${ }^{1}$, Syunji Mizunoe 3 , Yasuhiro Matsuoka ${ }^{4}$, Jun-ichi Kadota ${ }^{3}$, \\ Nobuhiro Fujii ${ }^{2}$, Kazuhiro Nagata ${ }^{4}$ and Shigeru Kohno ${ }^{1}$
}

\begin{abstract}
Address: ${ }^{1}$ Second Department of Internal Medicine, Nagasaki University School of Medicine, Nagasaki, Japan, ${ }^{2}$ Department of Microbiology, Sapporo Medical University School of Medicine, Sapporo, Japan, ${ }^{3}$ Division of Pathogenesis and Disease Control, Department of Infectious Diseases, Oita University Faculty of Medicine, Oita, Japan and ${ }^{4}$ Department of Molecular and Cellular Biology and CREST/JST, Institute for Frontier Medical Sciences, Kyoto University, Japan

Email: Tomoyuki Kakugawa - tomoyukikakugawa@yahoo.co.jp; Shin-ichi Yokota - syokota@sapmed.ac.jp;

Hiroshi Mukae* - hmukae@net.nagasaki-u.ac.jp; Hiroshi Kubota - hkubota@frontier.kyoto-u.ac.jp; Noriho Sakamoto - nsakamot@nagasakiu.ac.jp; Syunji Mizunoe - MIZUNOE@med.oita-u.ac.jp; Yasuhiro Matsuoka - koto-koto@ny.thn.ne.jp; Jun-ichi Kadota - kadota@med.oitau.ac.jp; Nobuhiro Fujii - fujii@sapmed.ac.jp; Kazuhiro Nagata - nagata@ frontier.kyoto-u.ac.jp; Shigeru Kohno - s-kohno@net.nagasaki-u.ac.jp

* Corresponding author
\end{abstract}

Published: 4 November 2008

BMC Pulmonary Medicine 2008, 8:23 doi:10.1 I86/147I-2466-8-23

This article is available from: http://www.biomedcentral.com/I47I-2466/8/23

(c) 2008 Kakugawa et al; licensee BioMed Central Ltd.

This is an Open Access article distributed under the terms of the Creative Commons Attribution License (http://creativecommons.org/licenses/by/2.0), which permits unrestricted use, distribution, and reproduction in any medium, provided the original work is properly cited.

\begin{abstract}
Background: The pathological diagnosis of idiopathic interstitial pneumonias (IIP) by surgical lung biopsy is important for clinical decision-making. However, there is a need to use less invasive biomarkers to differentiate nonspecific interstitial pneumonia (NSIP) from other IIP such as usual interstitial pneumonia (UIP). Heat shock protein (HSP) 47, a collagen-specific molecular chaperone, is involved in the processing and/or secretion of procollagen. HSP47 is increased in various fibrotic diseases. We investigated the autoantibodies to HSP47 in IIPs.
\end{abstract}

Methods: We measured the serum levels of the autoantibodies to HSP47 in 38 patients with various forms of IIP [ 16 with idiopathic pulmonary fibrosis (IPF), I5 with idiopathic NSIP, 7 with cryptogenic organizing pneumonia (COP)] and 18 healthy volunteers.

Results: The serum levels of autoantibodies to HSP47 in patients with idiopathic NSIP were significantly higher than in patients with IPF $(P<0.01)$, COP $(P<0.05)$, and healthy volunteers $(P$ $<0.05$ ). In addition, those in fibrosing NSIP were significantly higher than those of cellular and fibrosing NSIP $(p<0.05)$.

Conclusion: We found high levels of anti-HSP47 autoantibody titers in sera of patients with idiopathic fibrosing NSIP compared with other IIPs and healthy volunteers.

\section{Background}

The classification of idiopathic interstitial pneumonias

(IIP) includes seven clinico-radiologic-pathological enti- ties. Usual interstitial pneumonia (UIP) and nonspecific interstitial pneumonia (NSIP) are the two largest subsets of IIP $[1,2]$. The distinction between NSIP and UIP is 
important for clinical decision-making because the prognosis is generally better and the response to corticosteroids and immunosuppressants is also better in patients with NSIP compared with UIP [3-7]. In addition, patients with cellular NSIP are reported to have excellent longterm prognosis, while the majority of patients with fibrotic NSIP die mostly within 5 to 10 years of diagnosis [6]. Because of these reasons, the distinction between cellular NSIP and fibrotic NSIP is also important.

Clinicians often speculate on the presence of such pathological changes based on noninvasive imaging studies such as high-resolution computed tomography (HRCT) scans. However, the discrimination between NSIP and UIP cannot always be predicted accurately by HRCT. Although surgical (open or thoracoscopic) lung biopsy has been traditionally the "gold standard" for the diagnosis of interstitial lung diseases (ILD) and is clinically relevant for selection of appropriate therapy [8], it seems to be relatively invasive examination especially for patients with advanced ILD. Accordingly, less invasive biomarkers that distinguish NSIP from other types of IIP should be developed.

Heat shock protein (HSP) 47 is a collagen-binding, stressinducible protein localized in the endoplasmic reticulum and is never released into the extracellular matrix. HSP47 has a specific role only in the intracellular processing of procollagen production as a collagen-specific molecular chaperone [9-12]. HSP47 expression is upregulated in animals with experimentally-induced fibrosis, including murine bleomycin-induced pulmonary fibrosis $[13,14]$, rat peritoneal screlosis [15] and carbon tetrachlorideinduced rat liver cirrhosis [16]. In addition, we reported previously that there was also increased expression of human HSP47 in the fibrotic lesions of idiopathic pulmonary fibrosis (IPF) $[17,18]$, fibrotic transplanted kidney [19], and peritoneal sclerosis [20]. Recent reports have demonstrated that HSP47 expression is highly tissue- and cell-specific, restricted to mostly phenotypically altered collagen-producing cells, and correlates well with that of collagen $[13,17-20]$. These findings suggest the important role of HSP47 in collagen synthesis in various fibrotic disorders.

HSP47 is also identified as an autoantigen in the sera of several rheumatoid arthritis (RA) patients $[21,22]$. Higher levels of HSP47 protein and autoantibodies to HSP47 in sera were also found in patients with the rheumatic autoimmune diseases, especially mixed connective tissue disease (MCTD) [23]. Despite these observations, little is known about the relationship between fibrotic interstitial lung diseases and autoantibodies to HSP47.
We hypothesized that autoantibody titers to HSP47 in sera are different in idiopathic UIP, idiopathic NSIP, COP and healthy subjects.

\section{Methods \\ Study populations}

The subjects of this study were all the patients admitted to the hospitals of Nagasaki University School of Medicine and Oita University Faculty of Medicine from April 1997 to March 2004 in whom the diagnosis of interstitial pneumonia was confirmed pathologically, and 18 healthy adult volunteers. This is a retrospective study. The patients included 16 patients with IPF (UIP), 15 with idiopathic NSIP, 7 with cryptogenic organizing pneumonia (COP). The diagnosis of UIP and NSIP was confirmed pathologically by open lung biopsy or video-assisted thoracoscopic surgery (VATS) and classified according to the American Thoracic Society/European Respiratory Society consensus criteria [1]. Idiopathic NSIP patients included 7 with cellular and fibrosing pattern and 8 with fibrosing pattern [1]. The diagnosis of COP was established histopathologically by VATS in one patient and by transbronchial lung biopsy in 6 patients. Sera were obtained from these patients within one month before lung biopsy. Patients with fibrotic disease in any organ other than pulmonary fibrosis were excluded from the study, and no fibrotic disease other than pulmonary fibrosis was detected in any patient during the study. These patients had neither signs nor positive serological and other markers of collagen vascular diseases. Patients with cancer in any organ and those suspected to have malignancy were excluded from the study. None of these patients had received steroids or other immunosuppressants at the time of clinical sample collection. Patients characteristics before lung biopsy including age, smoking history, results of pulmonary function tests and arterial blood gas analysis were collected from either the clinical notes or the records of their general practitioners. Sera were also obtained from 8 healthy male and 10 healthy female volunteers (median age 31, range 26-60). All healthy volunteers had normal chest radiographs, were free of symptoms and not taking any medications. The study protocol was approved by the institutional review board, and informed consent was obtained from the patients and healthy volunteers.

\section{Determination of autoantibody titers by ELISA}

Enzyme-linked immunosorbent assay (ELISA) for determination of autoantibodies to HSP47 was carried out essentially as described previously $[23,24]$. Briefly, recombinant HSP47 protein diluted at $1 \mu \mathrm{g} / \mathrm{ml}$ in $50 \mathrm{mM}$ sodium carbonate buffer ( $\mathrm{pH} 9.6$ ) was immobilized on a 96-well microplate. The wells were blocked with $2 \%$ bovine serum albumin (BSA) in phosphate-buffered saline (PBS) and then incubated with human sera diluted 100 -fold. Specific binding of serum IgG to HSP47 was 
detected by subsequent incubation of horseradish peroxidase-conjugated goat antibodies specific for the $\gamma$-chain of human IgG (BioSource, Camarillo, CA) and 3,3',5,5'tetramethylbenzidine solution. After terminating the reaction with equal volume of $1 \mathrm{M}$ phosphoric acid, absorbance at $450 \mathrm{~nm}$ was measured and used as an antibody titer.

\section{Statistical analysis}

All values were expressed as median (range). Differences among groups were examined using Kruskal-Wallis test. The post-hoc test used was Scheffe test. Statistical analysis was performed using StatView-J 5.0 software (Abacus Concepts; Berkeley, CA). A $p$ value $<0.05$ denoted the presence of a statistically significant difference.

\section{Results \\ Patient characteristics}

Table 1 shows the characteristics of patients enrolled in this study. The baseline demographic and physiologic characteristics were similar among the groups.

\section{Anti-HSP47 autoantibody titers in human sera}

HSP47-reactive IgG titers of patients with idiopathic NSIP (median, 0.281 [range, 0.194-0.734]) were significantly higher than those of idiopathic UIP (0.165 [0.059$0.361])(\mathrm{P}<0.01), \mathrm{COP}(0.137$ [0.101-0.394]) $(\mathrm{P}<$ $0.05)$, and healthy volunteers $(0.181[0.062-0.349])(\mathrm{P}<$ 0.05) (Fig. 1). HSP47-reactive IgG titers of patients with fibrosing NSIP $(0.367$ [0.206-0.734]) were significantly higher than those of the cellular and fibrosing NSIP (0.231 [0.194-0.395]) $(\mathrm{P}<0.05)($ Fig. 2). HSP47-reactive IgG titers of patients with cellular and fibrosing NSIP,
UIP, COP and healthy controls were not significantly different.

\section{Correlation between anti-HSP47 autoantibody titers in sera and clinical course and other markers}

Anti-HSP47 autoantibody titers did not correlate with survival or clinical data such as results of pulmonary function tests and arterial blood gas analysis (data not shown). Antibody titers to HSP47 also did not correlate with the serum levels of KL-6, surfactant protein (SP)-D and SP-A (data not shown).

\section{Discussion}

The present study of biopsy-proven cases clearly demonstrated that the anti-HSP47 titers of patients with idiopathic fibrosing NSIP were higher than those of patients with idiopathic UIP, idiopathic cellular and fibrosing NSIP, COP and healthy subjects. In contrast, the antiHSP47 autoantibody titers in sera of patients with idiopathic UIP and healthy controls were not different despite the fact that overexpression of HSP47 has been reported in fibrotic lesions of IPF patients $[17,18]$.

Our results suggest that anti-HSP47 autoantibody titers in sera might be useful to discriminate between idiopathic fibrosing NSIP and other types of IIP such as IPF. However, we do not recommend the use of this serum marker alone to differentiate idiopathic fibrosing NSIP and other types of IIP because (1) our study only included a relatively small number of patients, (2) there was some overlap in the serum levels of anti-HSP47 autoantibodies between UIP and NSIP, (3) this is a retrospective study. Moreover, the patients groups might not represent general

Table I: Patient characteristics

\begin{tabular}{|c|c|c|c|c|c|}
\hline & COP & Idiopathic UIP & Idiopathic cellular and fibrosing NSIP & Idiopathic fibrosing NSIP & $P$ value \\
\hline Age (years) & $68(43-79)$ & $64(34-75)$ & 7I (56-75) & $50(28-67)$ & ns \\
\hline Sex (male/female) & $5 / 2$ & $1 \mathrm{I} / 5$ & $2 / 5$ & $4 / 4$ & ns \\
\hline Smoking (none/ex/smoker) & $3 / 3 / 1$ & $6 / 4 / 6$ & $5 / 1 / 1$ & $4 / 2 / 2$ & ns \\
\hline \multicolumn{6}{|l|}{ Spirometry: } \\
\hline VC $(L)$ & $2.79(1.86-4.21)$ & $2.73(1.14-3.60)$ & $2.44(1.28-3.32)$ & $2.72(1.73-3.39)$ & ns \\
\hline predicted VC (\%) & $101.6(53.0-111.2)$ & $82.9(43.3-112.5)$ & $76.6(56.8-108.1)$ & 94.7 (66.9-101.5) & ns \\
\hline FEVI (L) & $2.17(1.39-2.81)$ & $2.18(1.04-2.67)$ & $1.73(0.99-2.53)$ & $2.2(1.26-2.98)$ & ns \\
\hline predicted FEV (\%) & $77.6(69.8-80.0)$ & $83.3(69.4-98.2)$ & $85.0(60.1-96.2)$ & $80.9(66.5-89.5)$ & ns \\
\hline \multicolumn{6}{|l|}{ Gas exchange: } \\
\hline DLco $(\mathrm{ml} / \mathrm{min} / \mathrm{mmHg})$ & $10.28(6.39-17.81)$ & $8.59(2.32-14.65)$ & $9.94(7.24-14.60)$ & $12.18(7.77-19.85)$ & ns \\
\hline predicted DLco (\%) & $58.6(49.3-105.1)$ & $46.3(14.2-97.3)$ & $67.5(44.7-109.3)$ & $53.1(46.8-71.9)$ & ns \\
\hline \multicolumn{6}{|l|}{ Lung volume: } \\
\hline predicted TLC (\%) & $88.3(49.0-98.4)$ & $69.3(32.6-78.7)$ & $68.3(66.3-104.2)$ & $74.8(62.9-96.6)$ & ns \\
\hline TLC (L) & $3.4 \mathrm{I}(2.32-5.8 \mathrm{I})$ & $3.86(1.27-4.78)$ & $4.24(2.27-8.80)$ & $3.15(2.44-4.46)$ & ns \\
\hline \multicolumn{6}{|l|}{ Arterial blood gases: } \\
\hline $\mathrm{PaO}_{2}(\mathrm{mmHg})$ & $76.2(61.3-83.1)$ & $80.7(47.2-103.3)$ & $74.2(70.0-86.4)$ & $85.4(77.5-92.9)$ & ns \\
\hline
\end{tabular}

Data are median (range). ns = not significant

$\mathrm{COP}=$ cryptogenic organizing pneumonia; $\mathrm{DLco}=$ diffusing capacity for carbon monoxide; $\mathrm{FEV}=$ forced expiratory volume; NSIP = nonspecific interstitial pneumonia; TLC = total lung capacity; UIP = usual interstitial pneumonia; VC = vital capacity. 


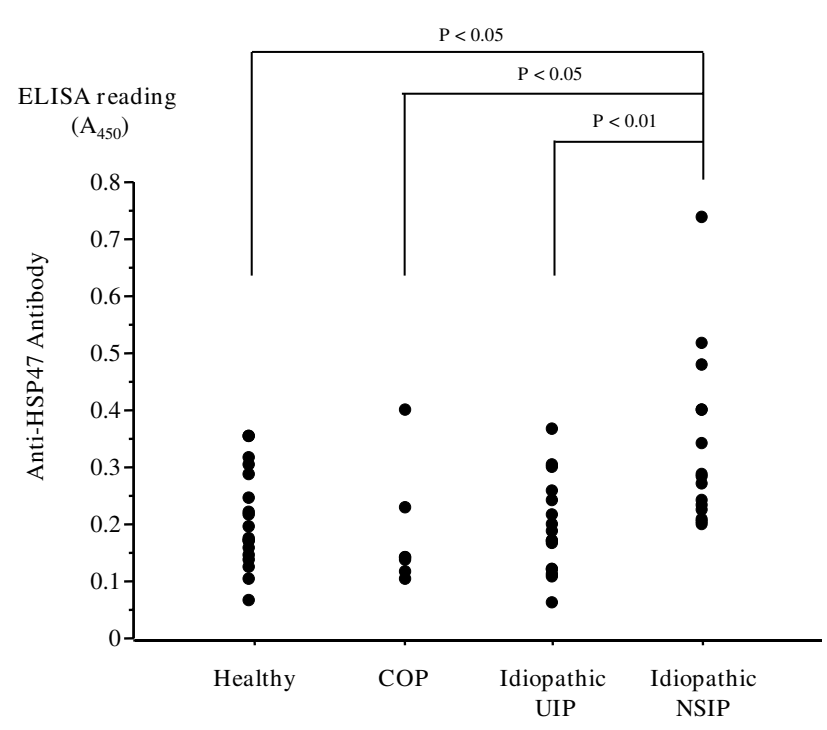

Figure I

Scattergram of IgG titers to HSP47 in patients with cryptogenic organizing pneumonia (COP), idiopathic usual interstitial pneumonia (UIP), idiopathic nonspecific interstitial pneumonia (NSIP) and healthy volunteer. Antibody titers are expressed as absorbance at $450 \mathrm{~nm}$.

patient population with IIP because the diagnosis of UIP and NSIP was confirmed pathologically by open lung biopsy or video-assisted thoracoscopic surgery and patients diagnosed only clinically were excluded. Although surgical lung biopsy has been traditionally the "gold standard" for the diagnosis of ILD, it seems to be relatively invasive examination especially for patients with advanced ILD. Accordingly, the study population may represent only "early and moderate" stage of ILD. Wellplanned prospective study using a large number of patients are required to determine the cutoff levels of antiHSP47 autoantibody titers necessary for the diagnosis, together with analysis of the sensitivity and specificity of such levels.

In the present study, we also investigated whether serum titers of anti-HSP47 autoantibody correlated with clinical course and other serum markers. However, anti-HSP47 autoantibody titers did not correlate with survival or clinical data such as results of pulmonary function tests and arterial blood gas analysis (data not shown). This is probably because of the small sample size. In addition, the titer of HSP47 antibody did not correlate significantly with the serum levels of KL-6, SP-D and SP-A, which were previously reported to be correlated with clinical activity of interstitial pneumonia [25-27], probably because of differences in the origins of these markers.
Why were the serum levels of autoantibody to HSP47 elevated in idiopathic NSIP patients, but not in idiopathic UIP patients, while fibrotic changes and expression of HSP47 in the lungs of patients with idiopathic UIP are much more severe than those with NSIP [18]? HSP47 is usually never released into the extracellular matrix, so it appears that intracellular HSP47 protein leaks into the peripheral blood when there is inflammation leading to tissue destruction. Elevation of anti-HSP47 autoantibody titers in idiopathic fibrosing NSIP patients might be due to the distinctive characteristics of idiopathic fibrosing NSIP including a variable degree of inflammation and fibrosis within the alveolar walls, which may induce leakage of HSP47 protein into the peripheral blood and subsequently induce the production of anti-HSP47 autoantibody. In contrast, severe fibrosis is seen but inflammation is mild in idiopathic UIP. A previous report reported that MCTD patients had markedly high levels of both HSP47 protein and autoantibodies to HSP47 in the sera compared with other rheumatic autoimmune diseases, including rheumatoid arthritis, systemic lupus erythematosus, and Sjögren syndrome [23]. This also suggests that the combination of inflammation and fibrosis is necessary for HSP47 protein to leak into the peripheral blood. However, this theory does not account for the reason why HSP47-reactive IgG titers of patients with fibrosing NSIP were significantly higher than those of idiopathic cellular and fibrosing NSIP. Many of the patients with idiopathic NSIP develop collagen vascular disease later on in the disease course. The fact that the serum levels of autoantibodies to HSP47 in patients with idiopathic NSIP were significantly higher than in patients with other IIPs suggests that the patients with so called "idiopathic" NSIP might display signs of auto-immunity in contrast to patients with idiopathic UIP.

As we previously reported, expression of HSP47 was noted in fibroblasts, myofibroblasts and type II pneumocytes in idiopathic interstitial pneumonia $[17,18]$. The expression level of HSP47 in type II pneumocytes of idiopathic UIP was significantly higher than that in idiopathic NSIP [18]. In contrast to that, in this study, we demonstrated that the anti-HSP47 titers of patients with idiopathic NSIP were significantly higher than those of patients with idiopathic UIP. We speculate that HSP47 autoantibody might neutralize the HSP47 antigen and suppress the fibrosis in idiopathic NSIP. However, there is no direct evidence. Further studies are warranted in order to elucidate the precise mechanisms.

Nevertheless, our findings support the concept that these diseases are different pathophysiological entities with different fibrotic pathways. We speculate that evaluation of anti-HSP47 autoantibody titers might be a useful method 


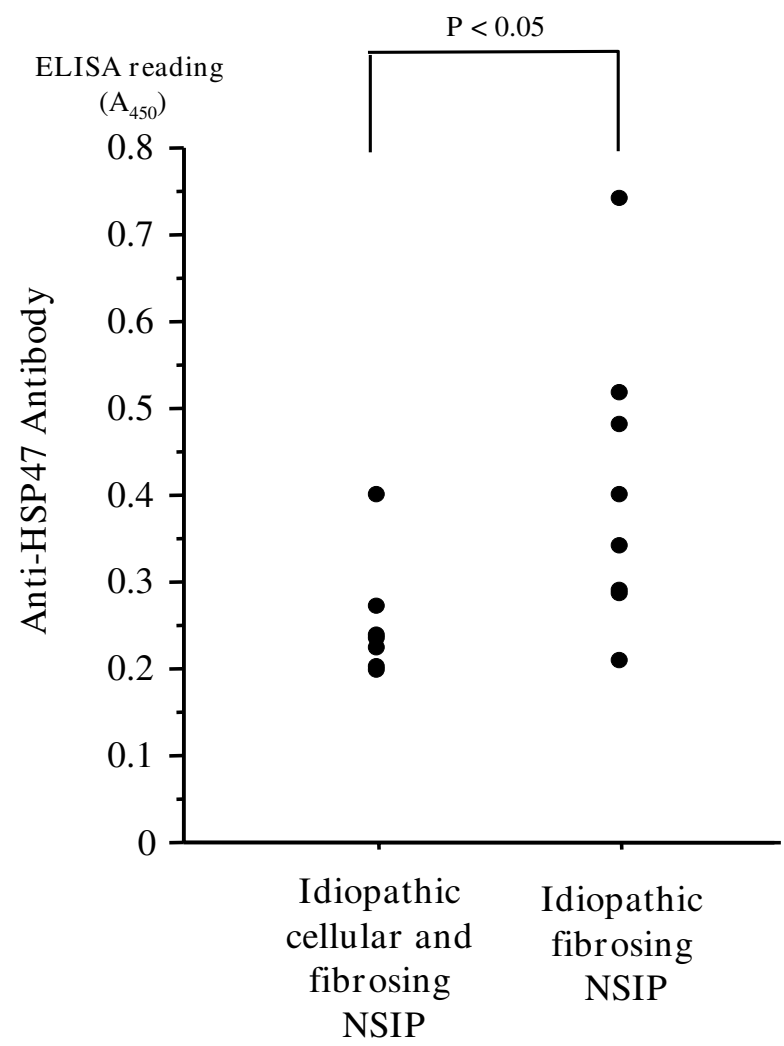

Figure 2

Scattergram of IgG titers to HSP47 in patients with idiopathic cellular and fibrosing nonspecific interstitial pneumonia (NSIP) and idiopathic fibrosing NSIP. Antibody titers are expressed as absorbance at $450 \mathrm{~nm}$.

to understand the differences in the underlying pathogenic mechanisms of these diseases.

\section{Conclusion}

In conclusion, we found high levels of anti-HSP47 autoantibody titers in sera of patients with idiopathic fibrosing NSIP compared with idiopathic UIP, idiopathic cellular and fibrosing NSIP, COP and healthy volunteers. However, further studies of a large number of patients are required to determine the prognostic and therapeutic values of anti-HSP47 autoantibody titers.

\section{Competing interests}

The authors declare that they have no competing interests.

\section{Authors' contributions}

All authors read and approved the final manuscript. TK, SY and HM have made substantial contributions to conception and design. TK and SY have made substantial contribution to acquisition and analysis of data. SY have made substantial contribution to determination of autoantibody titers by ELISA. HK and YM have made sub- stantial contribution to preparation of recombinant HSP47 protein. TK, SY, HM, NS, SM and JK have been involved in collecting clinical samples. TK, SY and HM have been involved in drafting the article. NS, NF, KN and SK have been involved in revising it critically for important intellectual content.

\section{Acknowledgements}

The authors thank Dr. M Kitaichi (Department of Laboratory Medicine and Pathology, NHO Kinki-chuo Chest Medical Center) for the valuable advice regarding pathological diagnosis. This study was supported in part by a research grant from the Ministry of Education, Science, Sports, and Culture of Japan.

\section{References}

I. American Thoracic Society/European Respiratory Society International Multidisciplinary Consensus Classification of the Idiopathic Interstitial Pneumonias. This joint statement of the American Thoracic Society (ATS), and the European Respiratory Society (ERS) was adopted by the ATS board of directors, June $200 \mathrm{I}$ and by the ERS Executive Committee, June 200 I. Am J Respir Crit Care Med 2002, 165(2):277-304.

2. Katzenstein AL, Myers JL: Idiopathic pulmonary fibrosis: clinical relevance of pathologic classification. Am J Respir Crit Care Med 1998, I57(4 Pt I):|30|-|3|5.

3. Bjoraker JA, Ryu JH, Edwin MK, Myers JL, Tazelaar HD, Schroeder DR, Offord KP: Prognostic significance of histopathologic subsets in idiopathic pulmonary fibrosis. Am J Respir Crit Care Med 1998, 157(I): 199-203.

4. Daniil ZD, Gilchrist FC, Nicholson AG, Hansell DM, Harris J, Colby TV, du Bois RM: A histologic pattern of nonspecific interstitial pneumonia is associated with a better prognosis than usual interstitial pneumonia in patients with cryptogenic fibrosing alveolitis. Am J Respir Crit Care Med 1999, 160(3):899-905.

5. Nicholson AG, Colby TV, du Bois RM, Hansell DM, Wells AU: The prognostic significance of the histologic pattern of interstitial pneumonia in patients presenting with the clinical entity of cryptogenic fibrosing alveolitis. Am J Respir Crit Care Med 2000, 162(6):2213-2217.

6. Travis WD, Matsui K, Moss J, Ferrans VJ: Idiopathic nonspecific interstitial pneumonia: prognostic significance of cellular and fibrosing patterns: survival comparison with usual interstitial pneumonia and desquamative interstitial pneumonia. Am J Surg Pathol 2000, 24(I): 19-33.

7. Flaherty KR, Toews GB, Travis WD, Colby TV, Kazerooni EA, Gross $\mathrm{BH}$, Jain A, Strawderman RL 3rd, Paine R, Flint A, Lynch JP 3rd, Martinez F): Clinical significance of histological classification of idiopathic interstitial pneumonia. Eur Respir J 2002, 19(2):275-283.

8. Hunninghake GW Zimmerman MB, Schwartz DA, King TE Jr, Lynch J, Hegele R, Waldron J, Colby T, Muller N, Lynch D, Galvin J, Gross B, Hogg J, Toews G, Helmers R, Cooper JA Jr, Baughman R, Strange C, Millard M: Utility of a lung biopsy for the diagnosis of idiopathic pulmonary fibrosis. Am J Respir Crit Care Med 200I, 164(2): 193-196.

9. Nakai A, Satoh M, Hirayoshi K, Nagata K: Involvement of the stress protein HSP47 in procollagen processing in the endoplasmic reticulum. J Cell Biol I992, I I 7(4):903-9|4.

10. Nagata K, Saga S, Yamada KM: A major collagen-binding protein of chick embryo fibroblasts is a novel heat shock protein. J Cell Biol 1986, I03(I):223-229.

II. Saga S, Nagata K, Chen WT, Yamada KM: pH-dependent function, purification, and intracellular location of a major collagenbinding glycoprotein. J Cell Biol 1987, I05(I):5 I7-527.

12. Sauk JJ, Smith T, Norris K, Ferreira L: Hsp47 and the translationtranslocation machinery cooperate in the production of alpha I(I) chains of type I procollagen. J Biol Chem 1994, 269(6):394I-3946.

13. Ishii H, Mukae H, Kakugawa T, Iwashita T, Kaida H, Fujii T, Hayashi T, Kadota J, Kohno S: Increased expression of collagen-binding heat shock protein 47 in murine bleomycin-induced pneumopathy. Am J Physiol Lung Cell Mol Physiol 2003, 285(4):L957-963. 
14. Kakugawa T, Mukae H, Hayashi T, Ishii H, Abe K, Fujii T, Oku H, Miyazaki M, Kadota J, Kohno S: Pirfenidone attenuates expression of HSP47 in murine bleomycin-induced pulmonary fibrosis. Eur Respir J 2004, 24(I):57-65.

15. Nishino T, Miyazaki M, Abe K, Furusu A, Mishima Y, Harada T, Ozono Y, Koji T, Kohno S: Antisense oligonucleotides against collagen-binding stress protein HSP47 suppress peritoneal fibrosis in rats. Kidney Int 2003, 64(3):887-896.

16. Masuda H, Fukumoto M, Hirayoshi K, Nagata K: Coexpression of the collagen-binding stress protein HSP47 gene and the alpha I(I) and alpha I (III) collagen genes in carbon tetrachloride-induced rat liver fibrosis. I Clin Invest 1994, 94(6):248I-2488.

17. Iwashita T, Kadota J, Naito S, Kaida H, Ishimatsu Y, Miyazaki M, Ozono Y, Kohno S: Involvement of collagen-binding heat shock protein 47 and procollagen type I synthesis in idiopathic pulmonary fibrosis: contribution of type II pneumocytes to fibrosis. Hum Pathol 2000, 3 I (I 2): | 498-I 505.

18. Kakugawa T, Mukae H, Hayashi T, Ishii H, Nakayama S, Sakamoto N, Yoshioka S, Sugiyama K, Mine M, Mizuta Y, Kohno S: Expression of HSP47 in usual interstitial pneumonia and nonspecific interstitial pneumonia. Respir Res 2005, 6:57.

19. Abe K, Ozono Y, Miyazaki M, Koji T, Shioshita K, Furusu A, Tsukasaki S, Matsuya F, Hosokawa N, Harada T, Taguchi T, Nagata K, Kohno S: Interstitial expression of heat shock protein 47 and alphasmooth muscle actin in renal allograft failure. Nephrol Dial Transplant 2000, I 5(4):529-535

20. Shioshita K, Miyazaki M, Ozono Y, Abe K, Taura K, Harada T, Koji T, Taguchi T, Kohno S: Expression of heat shock proteins 47 and 70 in the peritoneum of patients on continuous ambulatory peritoneal dialysis. Kidney Int 2000, 57(2):619-63I.

21. Hattori T, Fujisawa T, Sasaki K, Yutani Y, Nakanishi T, Takahashi K, Takigawa $M$ : Isolation and characterization of a rheumatoid arthritis-specific antigen (RA-A47) from a human chondrocytic cell line (HCS-2/8). Biochem Biophys Res Commun 1998, 245(3):679-683.

22. Hattori T, Takahash K, Yutani $Y$, Fujisawa T, Nakanishi T, Takigawa M: Rheumatoid arthritis-related antigen $47 \mathrm{kDa}$ (RA-A47) is a product of colligin-2 and acts as a human HSP47. J Bone Miner Metab 2000, I 8(6):328-334.

23. Yokota S, Kubota H, Matsuoka Y, Naitoh M, Hirata D, Minota S, Takahashi $H$, Fujii N, Nagata K: Prevalence of HSP47 antigen and autoantibodies to HSP47 in the sera of patients with mixed connective tissue disease. Biochem Biophys Res Commun 2003, 303(2):4|3-4|8.

24. Yokota SI, Hirata D, Minota S, Higashiyama T, Kurimoto M, Yanagi H, Yura T, Kubota H: Autoantibodies against chaperonin CCT in human sera with rheumatic autoimmune diseases: comparison with antibodies against other $\mathrm{Hsp} 60$ family proteins. Cell Stress Chaperones 2000, 5(4):337-346.

25. Ishii H, Mukae H, Kadota J, Kaida H, Nagata T, Abe K, Matsukura S, Kohno S: High serum concentrations of surfactant protein $A$ in usual interstitial pneumonia compared with non-specific interstitial pneumonia. Thorax 2003, 58(I):52-57.

26. Kobayashi J, Kitamura S: KL-6: a serum marker for interstitial pneumonia. Chest 1995, I08(2):311-315.

27. Takahashi H, Fujishima T, Koba H, Murakami S, Kurokawa K, Shibuya Y, Shiratori M, Kuroki Y, Abe S: Serum surfactant proteins A and $D$ as prognostic factors in idiopathic pulmonary fibrosis and their relationship to disease extent. Am J Respir Crit Care Med 2000, I62(3 Pt I): I |09-I I|4.

\section{Pre-publication history}

The pre-publication history for this paper can be accessed here:

http://www.biomedcentral.com/1471-2466/8/23/prepub
Publish with Bio Med Central and every scientist can read your work free of charge

"BioMed Central will be the most significant development for disseminating the results of biomedical research in our lifetime. "

Sir Paul Nurse, Cancer Research UK

Your research papers will be:

- available free of charge to the entire biomedical community

- peer reviewed and published immediately upon acceptance

- cited in PubMed and archived on PubMed Central

- yours - you keep the copyright
BioMedcentral 